\title{
Research: Articulating Questions, Generating Hypotheses, and Choosing Study Designs
}

Mary P Tully

\section{INTRODUCTION}

A rticulating a clear and concise research question is fundamental to conducting a robust and useful research study. Although "getting stuck into" the data collection is the exciting part of research, this preparation stage is crucial. Clear and concise research questions are needed for a number of reasons. Initially, they are needed to enable you to search the literature effectively. They will allow you to write clear aims and generate hypotheses. They will also ensure that you can select the most appropriate research design for your study.

This paper begins by describing the process of articulating clear and concise research questions, assuming that you have minimal experience. It then describes how to choose research questions that should be answered and how to generate study aims and hypotheses from your questions. Finally, it describes briefly how your question will help you to decide on the research design and methods best suited to answering it.

\section{TURNING CURIOSITY INTO QUESTIONS}

A research question has been described as "the uncertainty that the investigator wants to resolve by performing her study" or "a logical statement that progresses from what is known or believed to be true to that which is unknown and requires validation". ${ }^{2}$ Developing your question usually starts with having some general ideas about the areas within which you want to do your research. These might flow from your clinical work, for example. You might be interested in finding ways to improve the pharmaceutical care of patients on your wards. Alternatively, you might be interested in identifying the best antihypertensive agent for a particular subgroup of patients. Lipowski ${ }^{2}$ described in detail how work as a practising pharmacist can be used to great advantage to generate interesting research questions and hence useful research studies. Ideas could come from questioning received wisdom within your clinical area or the rationale behind quick fixes or workarounds, or from wanting to improve the quality, safety, or efficiency of working practice.

Alternatively, your ideas could come from searching the literature to answer a query from a colleague. Perhaps you could not find a published answer to the question you were asked, and so you want to conduct some research yourself. However, just searching the literature to generate questions is not to be recommended for novices - the volume of material can feel totally overwhelming.

Use a research notebook, where you regularly write ideas for research questions as you think of them during your clinical practice or after reading other research papers. It has been said that the best way to have a great idea is to have lots of ideas and then choose the best. The same would apply to research questions!

When you first identify your area of research interest, it is likely to be either too narrow or too broad. Narrow questions (such as "How is drug X prescribed for patients with condition $\mathrm{Y}$ in my hospital?") are usually of limited interest to anyone other than the researcher. Broad questions (such as "How can pharmacists provide better patient care?") must be broken down into smaller, more manageable questions. If you are interested in how pharmacists can provide better care, for example, you might start to narrow that topic down to how pharmacists can provide better care for one condition (such as affective disorders) for a particular subgroup of patients (such as teenagers). Then you could focus it even further by considering a specific disorder (depression) and a particular type of service that pharmacists could provide (improving patient adherence). At this stage, you could write your research question as, for example, "What role, if any, can pharmacists play in improving adherence to fluoxetine used for depression in teenagers?"

\section{TYPES OF RESEARCH QUESTIONS}

Being able to consider the type of research question that you have generated is particularly useful when deciding what research methods to use. There are 3 broad categories of question: descriptive, relational, and causal.

\section{Descriptive}

One of the most basic types of question is designed to ask systematically whether a phenomenon exists. For example, we 
could ask "Do pharmacists 'care' when they deliver pharmaceutical care?" This research would initially define the key terms (i.e., describing what "pharmaceutical care" and "care" are), and then the study would set out to look for the existence of care at the same time as pharmaceutical care was being delivered.

When you know that a phenomenon exists, you can then ask description and/or classification questions. The answers to these types of questions involve describing the characteristics of the phenomenon or creating typologies of variable subtypes. In the study above, for example, you could investigate the characteristics of the "care" that pharmacists provide. Classifications usually use mutually exclusive categories, so that various subtypes of the variable will have an unambiguous category to which they can be assigned. For example, a question could be asked as to "what is a pharmacist intervention" and a definition and classification system developed for use in further research.

When seeking further detail about your phenomenon, you might ask questions about its composition. These questions necessitate deconstructing a phenomenon (such as a behaviour) into its component parts. Within hospital pharmacy practice, you might be interested in asking questions about the composition of a new behavioural intervention to improve patient adherence, for example, "What is the detailed process that the pharmacist implicitly follows during delivery of this new intervention?"

\section{Relational}

After you have described your phenomena, you may then be interested in asking questions about the relationships between several phenomena. If you work on a renal ward, for example, you may be interested in looking at the relationship between hemoglobin levels and renal function, so your question would look something like this: "Are hemoglobin levels related to level of renal function?" Alternatively, you may have a categorical variable such as grade of doctor and be interested in the differences between them with regard to prescribing errors, so your research question would be "Do junior doctors make more prescribing errors than senior doctors?" Relational questions could also be asked within qualitative research, where a detailed understanding of the nature of the relationship between, for example, the gender and career aspirations of clinical pharmacists could be sought.

\section{Causal}

Once you have described your phenomena and have identified a relationship between them, you could ask about the causes of that relationship. You may be interested to know whether an intervention or some other activity has caused a change in your variable, and your research question would be about causality. For example, you may be interested in asking,
"Does captopril treatment reduce blood pressure?" Generally, however, if you ask a causality question about a medication or any other health care intervention, it ought to be rephrased as a causality-comparative question. Without comparing what happens in the presence of an intervention with what happens in the absence of the intervention, it is impossible to attribute causality to the intervention. Although a causality question would usually be answered using a comparative research design, asking a causality-comparative question makes the research design much more explicit. So the above question could be rephrased as, "Is captopril better than placebo at reducing blood pressure?"

The acronym PICO has been used to describe the components of well-crafted causality-comparative research questions. ${ }^{3}$ The letters in this acronym stand for Population, Intervention, Comparison, and Outcome. They remind the researcher that the research question should specify the type of participant to be recruited, the type of exposure involved, the type of control group with which participants are to be compared, and the type of outcome to be measured. Using the PICO approach, the above research question could be written as "Does captopril [intervention] decrease rates of cardiovascular events [outcome] in patients with essential hypertension [population] compared with patients receiving no treatment [comparison]?"

\section{DECIDING WHETHER TO ANSWER A RESEARCH QUESTION}

Just because a question can be asked does not mean that it needs to be answered. Not all research questions deserve to have time spent on them. One useful set of criteria is to ask whether your research question is feasible, interesting, novel, ethical, and relevant. ${ }^{1}$ The need for research to be ethical will be covered in a later paper in the series, so is not discussed here. The literature review is crucial to finding out whether the research question fulfils the remaining 4 criteria.

Conducting a comprehensive literature review will allow you to find out what is already known about the subject and any gaps that need further exploration. You may find that your research question has already been answered. However, that does not mean that you should abandon the question altogether. It may be necessary to confirm those findings using an alternative method or to translate them to another setting. If your research question has no novelty, however, and is not interesting or relevant to your peers or potential funders, you are probably better finding an alternative.

The literature will also help you learn about the research designs and methods that have been used previously and hence to decide whether your potential study is feasible. As a novice researcher, it is particularly important to ask if your planned study is feasible for you to conduct. Do you or your collabora- 
tors have the necessary technical expertise? Do you have the other resources that will be needed? If you are just starting out with research, it is likely that you will have a limited budget, in terms of both time and money. Therefore, even if the question is novel, interesting, and relevant, it may not be one that is feasible for you to answer.

\section{GENERATING AIMS AND HYPOTHESES}

All research studies should have at least one research question, and they should also have at least one aim. As a rule of thumb, a small research study should not have more than 2 aims as an absolute maximum. The aim of the study is a broad statement of intention and aspiration; it is the overall goal that you intend to achieve. The wording of this broad statement of intent is derived from the research question. If it is a descriptive research question, the aim will be, for example, "to investigate" or "to explore". If it is a relational research question, then the aim should state the phenomena being correlated, such as "to ascertain the impact of gender on career aspirations". If it is a causal research question, then the aim should include the direction of the relationship being tested, such as "to investigate whether captopril decreases rates of cardiovascular events in patients with essential hypertension, relative to patients receiving no treatment".

The hypothesis is a tentative prediction of the nature and direction of relationships between sets of data, phrased as a declarative statement. Therefore, hypotheses are really only required for studies that address relational or causal research questions. For the study above, the hypothesis being tested would be "Captopril decreases rates of cardiovascular events in patients with essential hypertension, relative to patients receiving no treatment". Studies that seek to answer descriptive research questions do not test hypotheses, but they can be used for hypothesis generation. Those hypotheses would then be tested in subsequent studies.

\section{CHOOSING THE STUDY DESIGN}

The research question is paramount in deciding what research design and methods you are going to use. There are no inherently bad research designs. The rightness or wrongness of the decision about the research design is based simply on whether it is suitable for answering the research question that you have posed.

It is possible to select completely the wrong research design to answer a specific question. For example, you may want to answer one of the research questions outlined above: "Do pharmacists 'care' when they deliver pharmaceutical care?" Although a randomized controlled study is considered by many as a "gold standard" research design, such a study would just not be capable of generating data to answer the question posed.
Similarly, if your question was, "Is captopril better than placebo at reducing blood pressure?", conducting a series of in-depth qualitative interviews would be equally incapable of generating the necessary data. However, if these designs are swapped around, we have 2 combinations (pharmaceutical care investigated using interviews; captopril investigated using a randomized controlled study) that are more likely to produce robust answers to the questions.

The language of the research question can be helpful in deciding what research design and methods to use. Subsequent papers in this series will cover these topics in detail. For example, if the question starts with "how many" or "how often", it is probably a descriptive question to assess the prevalence or incidence of a phenomenon. An epidemiological research design would be appropriate, perhaps using a postal survey or structured interviews to collect the data. If the question starts with "why" or "how", then it is a descriptive question to gain an in-depth understanding of a phenomenon. A qualitative research design, using in-depth interviews or focus groups, would collect the data needed. Finally, the term "what is the impact of" suggests a causal question, which would require comparison of data collected with and without the intervention (i.e., a before-after or randomized controlled study).

\section{CONCLUSIONS}

This paper has briefly outlined how to articulate research questions, formulate your aims, and choose your research methods. It is crucial to realize that articulating a good research question involves considerable iteration through the stages described above. It is very common that the first research question generated bears little resemblance to the final question used in the study. The language is changed several times, for example, because the first question turned out not to be feasible and the second question was a descriptive question when what was really wanted was a causality question. The books listed in the "Further Reading" section provide greater detail on the material described here, as well as a wealth of other information to ensure that your first foray into conducting research is successful.

\section{References}

1. Hulley S, Cummings S, Browner W, Grady D, Newman T. Designing clinical research. 4th ed. Philadelphia (PA): Lippincott, Williams and Wilkins; 2013.

2. Lipowski EE. Developing great research questions. Am J Health Syst Pharm. 2008;65(17):1667-70.

3. Richardson WS, Wilson MC, Nishikawa J, Hayward RS. The well-built clinical question: a key to evidence-based decisions. ACP J Club. 1995; 123(3):A12-3.

\section{Further Reading}

Cresswell J. Research design: qualitative, quantitative and mixed methods approaches. London (UK): Sage; 2009.

Haynes RB, Sackett DL, Guyatt GH, Tugwell P. Clinical epidemiology: how to do clinical practice research. 3rd ed. Philadelphia (PA): Lippincott, Williams \& Wilkins; 2006. 
Kumar R. Research methodology: a step-by-step guide for beginners. 3rd ed. London (UK): Sage; 2010

Smith FJ. Conducting your pharmacy practice research project. London (UK): Pharmaceutical Press; 2005.

Mary $\mathbf{P}$ Tully, PhD, is a Clinical Reader in Pharmacy Practice, Manchester Pharmacy School, University of Manchester, Manchester, United Kingdom.

Competing interests: Mary Tully has received personal fees from the UK Renal Pharmacy Group to present a conference workshop on writing research questions and nonfinancial support (in the form of travel and accommodation) from the Dubai International Pharmaceuticals and Technologies Conference and Exhibition (DUPHAT) to present a workshop on conducting pharmacy practice research.

\section{Address correspondence to:}

Dr Mary P Tully

Manchester Pharmacy School

University of Manchester

Oxford Road

Manchester M13 9PT UK

e-mail: Mary.tully@manchester.ac.uk

This article is the second in the CJHP Research Primer Series, an initiative of the CJHP Editorial Board and the CSHP Research Committee. The planned 2-year series is intended to appeal to relatively inexperienced researchers, with the goal of building research capacity among practising pharmacists. The articles, presenting simple but rigorous guidance to encourage and support novice researchers, are being solicited from authors with appropriate expertise.

Previous article in this series:

Bond CM. The research jigsaw: how to get started. Can J Hosp Pharm. 2014;67(1):28-30.

\section{CISADL 2013}

\section{Order your copy of the 2013 Canadian Investigational \& Special Access Drug List today!}

\section{Please use the CSHP order form found online at CSHP.ca to place your order.}

The CISADL is a compilation of drugs that are currently not marketed in Canada and may have Investigational or Special Access status with Health Canada. This list is not endorsed by Health Canada's Special Access Program. For precise information about the regulatory status of a drug, contact the Special Access Program directly. Entries to the list are contributed to by several Drug Information Centers across Canada and by Canadian drug manufacturers. In cases where non-marketed drugs do not appear on the list, foreign references should be consulted. The content of the list includes generic name and strength/concentration, dosage form, pharmacology/therapeutic use, trade and code name, source (manufacturer/distributor), and comments. Available in English only.

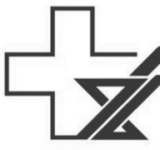
Société canadienne des pharmaciens d'hôpitaux 